\section{Constraint-induced movement therapy for focal hand dystonia in musicians}

Victor Candia, Thomas Elbert, Eckart Altenmüller, Harald Rau, Thomas Schäfer, Edward Taub

Focal hand dystonia is a condition involving manual incoordination that occurs in individuals, including musicians, who engage in extensive and foreceful use of the digits. To date, no treatments have been found to be effective. We found, with a non-invasive neuroimaging technique (magnetic source imaging), that musicians with focal hand dystonia exhibit a use-dependent overlap or smearing of the representational zones of the digits of the dystonic hand in the somatosensory cortex. ${ }^{1}$ Digital overuse had previously been found to produce a similar phenomenon in monkeys. ${ }^{2}$ Since behavioural mechanisms apparently underlie both the cortical disorder and the involuntary incoordination of movement, it was thought to be possible that a behavioural intervention could be of value in reducing or eliminating these conditions. The procedures employed in the present treatment approach are derived in part from a therapy, termed constraint-induced (CI) movement therapy, developed by Taub and co-workers that has been found to be effective in substantially improving the rehabilitation of upper extremity movement in stroke patients. ${ }^{3}$

Five professional musicians (three pianists and two guitarists) with long-standing symptoms were studied; they had previously received several treatments without success. The current therapy involved immobilisation by splint(s) of one or more of the digits other than the focal dystonic finger. The focal dystonic finger was required to carry out repetitive exercises in coordination with one or more of the other digits for $1.5-2.5 \mathrm{~h}$ daily (depending on patient fatigue) over a period of 8 consecutive days under therapist supervision. The patients subsequently continued practising the exercises with the splint for $1 \mathrm{~h}$ a day at home in combination with progressively longer periods of repertoire practice without the splint. Patient status was quantified with two measurement instruments: a dexterity/displacement device which continuously recorded digital displacement during metronome-paced movements of two fingers (spectral analysis of the records provided information concerning the smoothness of the movements before, during, and after training); and a dystonia evaluation scale (DES) in which the patients rated how well they were performing (without the

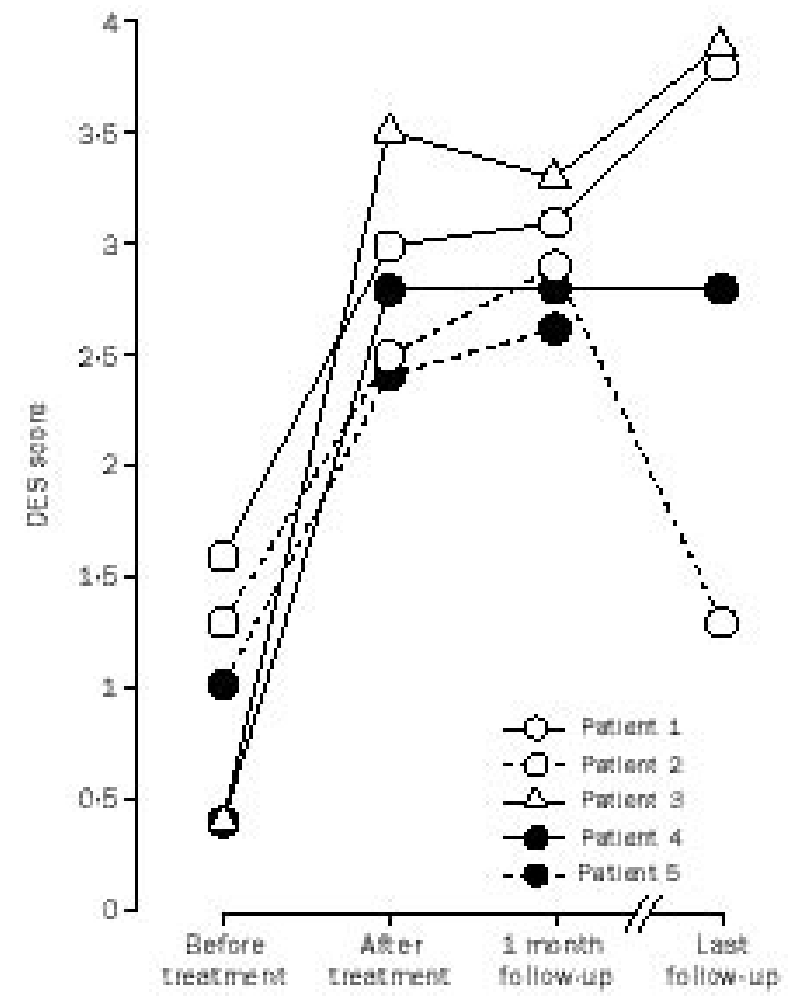

DES scores before and after treatment in five patients

splint) movement sequences and passages from their repertoire that had tended to generate dystonic movements in the past.

All patients showed improvement without the splint at the end of treatment. The DES scores are given in the figure. The ratings on the scale range from: $0=$ dystonia as bad as at its worst, $1=$ slightly improved, $2=$ moderately improved, $3=$ almost normal, and $4=$ normal. A one-way analysis of variance followed by post-hoc Scheffé tests revealed that pretreatment scores were different from post-treatment $(\mathrm{t}[4]=5 \cdot 2, \mathrm{p}<0 \cdot 01)$ and 1 -month follow-up $(\mathrm{t}[4]=7 \cdot 2$, $\mathrm{p}<0 \cdot 01)$ scores. The results on the DES were confirmed by the clinical evaluation of the treating neurologist and by the findings from the dexterity/displacement device obtained in the laboratory. The spectral power of the movements of the fingers of the dystonic hand in the band of the paced frequency showed an increase in the smoothness of those movements after treatment $(\mathrm{p}<0 \cdot 01)$. After treatment, the one patient who was non-compliant regressed after 9 months; three patients showed additional improvement into the normal or almost normal range; and one showed no reduction in a substantial therapeutic effect. Two of the musicians have resumed concert performances.

This research was supported by the Deutsche Forschungsgemeinschaft and by a grant from the Rehabilitation Research Service of the Veterans Administration (B95-975R).

1 Elbert T, Candia V, Altenmuller E, et al. Alteration of digital representations in somatosensory cortex in focal hand dystonia. Neuroreport 1998; 9.

2 Byl NN, Merzenich MM, Jenkins WM. A primate genesis model for focal dystonia and repetitive strain injury: learning-induced dedifferentiation of the representation of the hand in the primary somatosensory cortex in adult monkeys. Neurology 1996; 47: 508-20.

3 Taub E, Miller NE, Novack TA, et al. Technique to improve motor deficit after stroke. Arch Phys Med Rehabil 1993; 74: 347-54.

Department of Psychology, University of Konstanz, Fach D25, D-78457 Konstanz, Germany (T Elbert); Institut für Musikphysiologie und Musiker-Medizin, Hochschule für Musik und Theater Hannover, Hannover, Germany; and Department of Psychology, University of Alabama at Birmingham, Birmingham, AL, USA 